\title{
Health professionals responding to men for safety (HERMES): feasibility of a general practice training intervention to improve the response to male patients who have experienced or perpetrated domestic violence and abuse
}

\author{
Emma Williamson ${ }^{1}$, Sue K. Jones ${ }^{2}$, Giulia Ferrari ${ }^{2}$, Thangam Debbonaire ${ }^{3}$, Gene Feder ${ }^{2}$ and Marianne Hester \\ ${ }^{1}$ Centre for Gender and Violence Research, University of Bristol, Bristol, UK \\ ${ }^{2}$ NIHR School for Primary Care Research, Academic Unit of Primary Care, School of Social and Community Medicine, \\ University of Bristol, Bristol, UK \\ ${ }^{3}$ RESPECT, London, UK
}

\begin{abstract}
Aim: To evaluate a training intervention for general practice-based doctors and nurses in terms of the identification, documentation, and referral of male patients experiencing or perpetrating domestic violence and abuse (DVA) in four general practices in the south west of England. Background: Research suggests that male victims and perpetrators of DVA present to primary care clinicians to seek support for their experiences. We know that the response of primary care clinicians to women patients experiencing DVA improves from training and the establishment of referral pathways to specialist DVA services. Method: The intervention consisted of a 2-h practice-based training. Outcome measures included: a pre-post, self-reported survey of staff practice; disclosures of DVA as documented in medical records pre-post (six months) intervention; semi-structured interviews with clinicians; and practice-level contact data collected by DVA specialist agencies. Results: Results show a significant increase in clinicians' self-reported preparedness to meet the needs of male patients experiencing or perpetrating DVA. There was a small increase in male patients identified within the medical records (6 pre- to 17 post-intervention) but only five of those patients made contact with a specialist DVA agency identified within the referral pathway. The training increased clinicians' confidence in responding to male patients affected by DVA. The increase in recorded identification of DVA male patients experiencing or perpetrating DVA was small and contact of those patients with a specialist DVA support service was negligible. We need to better understand male help seeking in relation to DVA, further develop interventions to increase identification of male patients experiencing or perpetrating DVA behaviours, and facilitate access to support services.
\end{abstract}

Key words: domestic violence; family violence; male; patient care; primary care; training

Received 15 April 2014; revised 30 June 2014; accepted 2 September 2014; first

published online 23 September 2014

Correspondence to: Dr Emma Williamson, University of Bristol, Centre for Gender and Violence Research, 8 Priory Road, Bristol BS8 1TZ, UK. Email: E.williamson@bristol.ac.uk

\section{Introduction}

Data from the Crime Survey of England and Wales suggests that $\sim 4 \%$ of men report experience of one or more incidents of partner abuse in the

(C) Cambridge University Press 2014 
last year (Britton, 2012) with 11\% reporting having experienced 'non-sexual partner abuse' since the age of 16 [Office for National Statistics (ONS), 2013]. It is important to note, however, that the measurement of the prevalence of domestic violence and abuse (DVA) in general populations surveys, with focus only on incidents of abuse rather than impact, may not be useful in the clinical setting (Williamson, 2012; Hester, 2013).

Focusing on evidence from a clinical context, male patients, as victims or perpetrators of DVA, seek help from health services but usually do not spontaneously disclose abuse. The British Crime Survey (Hoare and Jansson, 2008) reported that $18 \%$ of self-identified male victims of DVA reported seeking medical assistance. A UK survey of 175 male patients in four practices reported that $15 \%$ had experienced potentially abusive behaviours and $16 \%$ self-identified as perpetrators. In that study, the majority of male patients thought it was helpful for men to be asked about perpetration and experience of DVA and $73 \%$ said they would be comfortable discussing the issue with their general practitioner (GP) (Westmarland et al., 2004). A US study of service use by perpetrators of DVA (Coben and Friedman, 2002) reported that $42 \%$ of those arrested for DVA perpetration had seen their health provider in the six months before arrest. This is consistent with findings from the United Kingdom (Hester et al., 2006), where 32 (from 45) perpetrators on a DVA perpetrator programme reported having consulted their GP before the programme attendance. While the evidence above might suggest that general practice could be a useful setting in which to address male patients' experience and perpetration of DVA, the issue of men and DVA has received little attention over the years that may go some way to explaining why the issue is not higher on the clinical radar of practitioners (Hines and Douglas, 2009). Recent NICE guidelines [National Institute of Clinical Excellence (NICE) 2014] focusing on DVA and its impacts on all patient groups, has identified the need for clinicians to address the needs of male patients as both victims and perpetrators of abuse.

\section{Background}

A number of studies have identified and explored different barriers to effective clinician responses to
DVA, although much of this research has focused on responses to predominately female patients. The barriers identified within this research include: inadequate preparation and training (Gutmanis et al., 2007); perceived complexity of the issue in relation to perpetrators (Miller and Jaye, 2007); concerns about treating members of the same family where DVA might be an issue (Hegarty et al., 2008); a reported lack of information about appropriate referral agencies (Miller and Jaye, 2007); concerns about differentiating between victims and perpetrators (Kimberg, 2008); inconsistencies in the documentation of DVA within the medical record (Williamson, 2000; Miller et al., 2005); time constraints in terms of availability for training and within patient consultations (Gadomski et al., 2001; Gutmanis et al., 2007); a reported lack of confidence in knowing how to respond to DVA (Miller and Jaye, 2007); attitudinal and preconceived ideas that might be detrimental to effective practice (Lo Fo Wong et al., 2006); and uncertainty about the role of health clinicians in relation to DVA (Yeung et al., 2012).

When addressing the needs of male patients it is often the perceived need to differentiate between 'genuine' victims and perpetrators that raises concern for clinicians and creates a barrier to effective practice. Evidence collated from the National DVA helpline for male victims, run by the domestic violence charity RESPECT (RESPECT, 2010), indicates that of 1441 men who contacted the helpline between 2007-2010, helpline workers determined by the end of each call that only $56.5 \%$ of callers would be classified as victims of abuse. Of the remaining callers $13 \%$ were describing an unhappy relationship where abuse was not present; $1.6 \%$ talked about mutual violence; $16.3 \%$ described incidents where they had perpetrated abuse; and in $12.5 \%$ of cases the helpline worker, despite extensive training on the issue, was still not sure. This highlights how distinguishing between the role of victim and perpetrator is complex and understandably raises concerns for clinicians, and that it should be carried out by DVA experts rather than clinicians.

These barriers also highlight the need for training to give practitioners the tools to address DVA within their clinical practice. Different training models have been trialled that includes the development of core curriculum principles (Brandt et al., 2007), interventions aimed at 
improving the identification and management of DVA (Thompson et al., 2000), and focus group training approaches (Lo Fo Wong et al., 2006). A training and support intervention for female patients in UK general practice was tested in a cluster randomised trial of 48 practices: Identification and Referral to Improve Safety (IRIS) (Feder et al., 2011). The intervention, delivered by an advocate-educator based in a third-sector organisation, included two 2-h training sessions for doctors and nurses, a 1-h training session for administrative staff, a referral pathway for women who disclosed DVA, and feedback to practices. The IRIS trial found a threefold difference in the identification of women experiencing abuse and a sixfold difference in referrals to the specialist local DVA agencies between the intervention and control sites.

The aim of the HERMES study was to evaluate the feasibility of a training intervention for family practice extending the IRIS model to improve the response to male patients who had experienced or perpetrated DVA. The objectives were: (i) development of the intervention, (ii) delivery of the intervention to four general practices, and (iii) mixed method evaluation of its feasibility.

\section{Methods}

\section{Development of the intervention}

The intervention was developed through meetings between the research team, primary care, and specialist DVA professionals. The content was based on RESPECT training principles (RESPECT, 2013) and streamlined within the process defined by the IRIS model (Feder et al., 2011). The training included information about prevalence and associated presenting complaints of male patients experiencing or perpetrating DVA; appropriate responses and nature of the referral pathway; the acting out of enquiry about abuse; and a discussion of issues potentially impacting on men who have sex with men. The intervention used a flowchart with all the pertinent information from the training including prompts on how to ask about abuse, symptoms suggestive of abuse issues, and a clear referral pathway (Supplementary data 1). Unlike the IRIS intervention (Feder et al., 2011), HERMES did not include a direct referral pathway to a local specialist DVA advocate. Instead, HERMES clinicians were advised to refer male patients to the two national RESPECT helplines for victims and perpetrators respectively by giving patients a card with both helpline numbers, one on each side.

\section{Delivery of the intervention}

A total of four general practices in Bristol that had already received the IRIS training were given the 2-h HERMES session by a GP and DVA specialist, using the IRIS interactive adult learning method. Clinicians in each practice were provided with the HERMES flowchart and the two-sided referral information cards. A prompt to ask about DVA was linked in the electronic medical record to conditions associated with DVA, such as anxiety and depression. Reception and administrative staff were also offered a 1-h information session on the intervention. Clinicians were also offered follow-up training.

\section{Evaluation of feasibility}

A range of methods were used to evaluate the feasibility of the intervention. This included measuring change in clinicians' practice and behaviour through the PROVIDE Intervention Measure (PIM); data extracted pre- and post-intervention from the medical records of the participating practices; referral data from the third sector specialist agency identified within the referral pathway; and semi-structured interviews with participating clinicians. Each of these are outlined below.

(i) The PIM (Supplementary data 2 and 3) was developed from the validated PREMIS questionnaire (Ramsey et al., 2012), and edited down to include only questions about clinical practice and behaviours. As such, the version used within this study was not a validated measure. Practitioners undertaking the training were asked to complete it before the training and again after six months. Data from both time frames were entered into an Access database and analysed in STATA 12 . We computed a Wilcoxon signed-rank sum test of the differences in responses between pre- and post-PIM training, imposing no distributional assumptions on the differences (Armitage et al., 2002). We also report baseline and 
follow-up median scores, as well as a bootstrapped median of the differences (with 95\% confidence interval), computed by re-sampling observations 50 times.

(ii) DVA identification data were extracted from electronic medical records of HERMES practices using codes designating DVA, six months before and after the intervention. For the analysis we compared numbers of patients identified as experiencing or perpetrating DVA. Cases that were identified through the searches were individually checked to ensure that they related to DVA cases. This procedure was carried out by a member of the clinical team in the presence of a researcher in order to adhere to ethical concerns about accessing personal information without the consent of individual patients.

(iii) Referral data were collected from RESPECT, who run the two national helplines specified within the referral pathway. Helpline staff asked all callers from the study city if they had heard of the service from their general practice and, if so, which one. To ensure the counting of patients who chose to self-refer to local DVA services, two local services, one for male victims the other for male perpetrators were contacted and data were collected.

(iv) A total of seven semi-structured telephone interviews were conducted with HERMES clinicians who had completed a postintervention PIM measure and who agreed to take part. Analysis involved a thematic analysis of the transcribed interview transcripts. Limited summary data from these interviews is presented in this paper.

The study was approved by the South West research ethics committee (reference 10/H0106/22).

\section{Results}

\section{PIM survey}

A total of 25 out of 34 practitioners who attended the training completed the baseline PIM survey. Respondents were aged between 31 to 60 years (median 47, SD 7.7), 16 were female and nine were male. In all, 19 respondents were GPs, five practice nurses, and one respondent was a health advisor. Respondents had between 4 and
30 years' experience working in family practice (median 15, SD 8.1), and reported seeing between 21 and 300 patients a week (median 80, SD 56.2).

Respondents reported $0-10 \mathrm{~h}$ previous training on DVA (median 4, SD 2.9) that included watching a video $(n=2)$, completing a web-based programme $(n=2)$, attending a lecture or talk $(n=14)$, skills-based training $(n=6)$, medical classroom-based $(n=3)$, or clinic-based $(n=2)$ training. The majority of respondents $(n=19)$ had received training as part of the IRIS project (Feder et al., 2011). Previous training included information about women as victims of DVA $(n=24)$, heterosexual men as victims $(n=11)$, heterosexual men as perpetrators $(n=14)$, and men having sex with men as either victims or perpetrators of DVA $(n=6)$.

Comparison of pre- and post-intervention responses (14 respondents) suggests an improvement in perceived ability to manage situations where patients either disclosed, or the clinicians suspected, exposure to DVA. There were statistically significant improvements in perceived competence in responding to heterosexual, gay, or bisexual male patients, but no significant change in dealing with female patients who may have been victims, the exception being ability to respond to disclosure (Table 1). This is consistent with all participating practices being part of the IRIS programme, and having previous training in relation to women experiencing DVA.

\section{Recording of DVA identification}

DVA identified and documented in medical records across all four practices in the six months before and after the training is shown in Table 2. A total of six male patients were identified before the intervention as experiencing or perpetrating intimate domestic violence compared with 17 male patients during the post-intervention period [intimate partner violence (IPV) is identified here to differentiate from domestic abuse experienced from wider family members]. According to information from the medical records, four of the six male patients identified before the intervention were classified as perpetrators of IPV, one as a possible perpetrator of IPV, and one as a victim of wider family abuse (by his brother). Postintervention, 2 of the 17 patients were identified as victims of IPV, four as victims of wider DVA 
Table 1 Change in practitioners' self-reported ability to identify and respond to DVA

\begin{tabular}{|c|c|c|c|c|c|c|}
\hline Feel comfortable & $n$ & $\begin{array}{l}\text { Signrank } \\
\text { test } P \text {-value }\end{array}$ & $\begin{array}{l}\text { Baseline } \\
\text { median }\end{array}$ & $\begin{array}{l}\text { Follow-up } \\
\text { median }\end{array}$ & $\begin{array}{l}\text { Median of the } \\
\text { differences }\end{array}$ & $95 \% \mathrm{Cl}$ \\
\hline Asking female victim & 14 & 0.046 & 4 & 5 & 0 & {$[0-0.5]$} \\
\hline Responding to female victim & 14 & 0.008 & 4 & 5 & 0.5 & {$[0-1]$} \\
\hline Identifying female victim & 13 & 0.056 & 4 & 4 & 0 & {$[0-1]$} \\
\hline Referring female victim & 13 & 0.096 & 4 & 5 & 0 & {$[0-1]$} \\
\hline Asking heterosexual male victim & 14 & 0.003 & 3 & 4 & 1 & {$[0-1.5]$} \\
\hline Responding to heterosexual male victim & 14 & 0.023 & 4 & 4 & 1 & {$[0-1.5]$} \\
\hline Identifying heterosexual male victim & 13 & 0.004 & 2.5 & 4 & 1 & {$[0-2]$} \\
\hline Referring heterosexual male victim & 13 & 0.003 & 3 & 4 & 1 & {$[1-2]$} \\
\hline Asking heterosexual male perpetrator & 14 & 0.005 & 3 & 4 & 1 & {$[0-1]$} \\
\hline Responding to heterosexual male perpetrator & 14 & 0.002 & 3 & 4 & 1 & {$[1-2]$} \\
\hline Identifying heterosexual male perpetrator & 13 & 0.002 & 3 & 4 & 1 & {$[0-1]$} \\
\hline Referring heterosexual male perpetrator & 12 & 0.002 & 3 & 4 & 1 & {$[1-2]$} \\
\hline Asking gay and bisexual male victim & 14 & 0.005 & 3 & 4 & 1 & {$[0-2]$} \\
\hline Responding to gay and bisexual male victim & 14 & 0.002 & 2.5 & 4 & 1 & {$[1-2]$} \\
\hline Identifying gay and bisexual male victim & 13 & 0.002 & 2 & 4 & 1 & {$[1-2]$} \\
\hline Referring gay and bisexual male victim & 13 & 0.003 & 2 & 4 & 2 & {$[1-3]$} \\
\hline Asking gay and bisexual male perpetrator & 14 & 0.005 & 3 & 4 & 1 & {$[0-1]$} \\
\hline Responding to gay and bisexual male perpetrator & 14 & 0.001 & 2 & 4 & 1 & {$[1-2]$} \\
\hline Identifying gay and bisexual male perpetrator & 13 & 0.001 & 2 & 3 & 1 & {$[1-2]$} \\
\hline Referring gay and bisexual male perpetrator & 12 & 0.003 & 2 & 4 & 2 & {$[1-3]$} \\
\hline
\end{tabular}

This table reports number of observations; the non-parametric signed-rank sum test of differences in the distributions between baseline and follow-up measurements; baseline and follow-up medians; and the bootstrapped median of the differences with its $95 \%$ confidence interval (CI) (the median of the differences and its interval are bootstrapped, ie, calculated by repeatedly sampling observations a number of times, in this case 50 ).

Table 2 Number of male patients documented within the medical record as experiencing or perpetrating DVA

\begin{tabular}{llllllll}
\hline Six-months period & \multicolumn{2}{l}{ Perpetrators } & & & \multicolumn{2}{l}{ Victims } \\
\cline { 2 - 4 } & Definite & Possible & All & & Definite & Possible \\
\hline Pre-intervention & 4 & 1 & 5 & 0 & 1 & All \\
Post-intervention & 7 & 4 & 11 & $6^{\text {a }}$ & 1 \\
\hline
\end{tabular}

DVA = domestic violence and abuse.

${ }^{\text {a }}$ Four of these six victims were victims of DVA within the wider family context and not DVA within an intimate partnership.

including other family members. In all, seven men were clearly identified as perpetrators of IPV and four male patients were identified as possible perpetrators of IPV.

\section{Contact with RESPECT helplines and local services}

RESPECT, who run helplines for male victims and perpetrators of DVA identified three referrals to their helplines via HERMES practices during the data monitoring period. Other patients could have accessed other help offered by the service through their website. Direct contact had been made to the local domestic violence perpetrator programme from two patients from the study practices. This resulted in a total of five patients, from HERMES active practices, contacting support services following the intervention.

\section{Interviews with practitioners}

Views on domestic violence and male patients

When discussing male patients as perpetrators or victims of DVA, informants claimed this was 
not a common problem for their patients. As one female doctor stated:

I think the training was fine [...] we just don't seem to see it as much, the sort of male issues. Whether that's because it's not as common or whether they're just not coming, [...] People just don't pitch up, even though my radar you know is more aware than it was before, it just doesn't seem in practice to be as common (EW03).

Another caveat to HERMES raised by some clinicians was that they tended to see mostly female patients (EW05), or predominantly older patients that one informant perceived as not being at risk of experiencing or perpetrating DVA. In both these cases there was an assumption that, while it was useful to be aware of the issues raised in the HERMES training this was unlikely to be a regular aspect of their work. However, there is no research evidence that age is a protective factor for older patients experiencing DVA.

Also evident was an assumption, when discussing male patients who might be victims of abuse, that this would more likely affect men who have sex with men. When asked about men in general, three of the seven clinicians interviewed responded by referring to gay men. These clinicians were equating male victims of abuse with gay men despite this not being the focus of the questions or the HERMES training. While it is positive that practitioners recognise that abuse can occur in men who have sex with men relationships (Hester et al., 2012; Donovan and Hester, 2014), this becomes problematic if there is an underlying assumption that the impact of DVA on men is limited to gay men.

\section{Safety concerns}

One of the issues that arose in interviews and is reported in the wider literature discussed above, is a concern about the safety of clinicians themselves.

I suppose it would only be you know I wouldn't feel particularly safe raising it with someone that was coming across aggressive. You know so I would choose not to, you know, and we do ha- we do have one patient at the moment that's very anti female doctors (laugh). So, there's nothing that I can think of inherent in me that would be a barrier.
It would be more whether I would choose not to on safety grounds if say a potential perpetrator was perpetrating (laugh) in the room (Female doctor, EW03).

Addressing the safety concerns of clinicians is crucial in enabling them to raise issues about anger and violence with patients who might then direct that aggression towards them. Removing a requirement for clinicians to differentiate between victims and perpetrators helped, but did not eliminate these concerns completely.

\section{Differentiations in the use of codes}

During the collection of DVA disclosure data from individual patient records it became apparent that different clinicians used abuse codes differently. We asked clinicians about how they used DVA codes in medical records and their responses support the case for more research into practitioners' current coding practices. As one doctor stated: 'I probably don't code it if I'm perfectly honest. I probably free text it in to the problem heading' (EW01). This raises concerns about how inconsistencies in documentation might be impacting on clinical practice and research (Miller et al., 2005).

\section{Time for training}

Most clinicians felt that the HERMES training would preferably be incorporated in a comprehensive package that also includes training on women experiencing DVA, specifically within the IRIS programme. While this preference was dictated often by concerns about time constraints, it also raises questions about how best to integrate training on tackling DVA with all patient groups.

Clinicians also highlighted the need for top-up training. 'I think that in order for the message to stick, we need to be reminded regularly' (SJ01). Both HERMES and the IRIS intervention had offered top-up training; however, this was not always practical to arrange, given the competing training demands of busy practitioners.

\section{Conclusions}

The findings from this pilot suggest that an intervention focused on the issue of DVA and male patients resulted in a number of positive outcomes. This pilot supports previous evidence from studies 
of female patients (McCaw et al., 2001; Lo Fo Wong et al., 2006; Gutmanis et al., 2007; Feder et al., 2011) that when provided with the relevant tools, through training, clinicians felt more prepared to address the needs of male patients experiencing or perpetrating DVA. Although the number of men identified from the medical records was low $(n=20)$, there was an increase in documentation at the post-intervention point, which is a positive outcome. An examination of the specific cases within the medical records data revealed some interesting examples of good practice where clinicians followed up initial discussions at subsequent appointments asking whether things were any better, or if the patient had accessed the specialist support. We also identified cases of male patients disclosing their concerns about the impact of their behaviour on the wider family. The low number of male patients who contacted the referral and local organisations $(n=5)$ raises additional questions about whether they might benefit from a local advocate referral (as in IRIS Feder et al., 2011) or whether more research is needed to establish how male victims and perpetrators are accessing and using support services.

An important secondary outcome of the research was that following the HERMES training there was an increase in the identification and documentation of female victims of DVA during the post-intervention period despite this not being the focus of the intervention training. With regard to the barriers reported in the wider literature, this pilot was able to address, through the training, some of the concerns reported by practitioners. These included feeling adequately prepared to address the issue; feeling more comfortable knowing they did not have to differentiate between victims and perpetrators (although still being concerned about potential aggression from perpetrators); having knowledge about referral agencies; and feeling more confident to raise the issue with male patients. This pilot study highlighted other areas where concerns still remained for practitioners, in particular concerns about how to manage abuse, particularly with respect to documentation, across patients in the same families.

There were a number of limitations to this pilot study that are important to recognise. As the study was a pilot, it included only a small number of general practices. The analysis of the PIM data tested across a wide range of outcomes that made it more likely for us to find statistically significant changes, although it should be noted that we found improvements in all areas targeted by the intervention. In relation to referral, our data were limited to the specialist agency named in the intervention, which meant that apart from the local services with whom we had contact, we were unable to measure if male patients had sought support elsewhere. Despite these limitations, however, the findings of the study support the hypothesis that participating clinicians benefited from the training.

Further research is needed to identify appropriate ways to document DVA for different members of the same family, and to ensure that attitudes towards sexuality and age do not prevent practitioners from engaging with all male patients who present with DVA related issues. We also need to better understand men's help-seeking behaviour in order to improve uptake of appropriate support services.

Finally, the key message from practitioners who participated in this pilot study was the need to consolidate DVA training about responding to male patients into existing DVA training packages. This was primarily owing to time constraints related to competing training needs. This raises some concerns about whether there is capacity, in terms of time, within existing training programmes to include the material needed to address the needs of male patients who may be experiencing and/or perpetrating DVA, was and is a barrier that researchers need to address in future interventions.

\section{Acknowledgements}

This paper presents independent research commissioned by the National Institute for Health Research (NIHR) under its Programme Grants for Applied Research scheme (RP-PG-0108-10084). The views expressed in this publication are those of the author(s) and not necessarily those of the NHS, the NIHR, or the Department of Health. The study was approved by the South West Research Ethics Committee (reference 10/H0106/22).

\section{Supplementary material}

To view supplementary material for this article, please visit http://dx.doi.org/10.1017/S1463423614000358

Primary Health Care Research \& Development 2015; 16: 281-288 


\section{References}

Armitage, P., Berry, G. and Matthews, J.N.S. 2002: Statistical methods in medical research. London: Blackwell Publishing.

Brandt, E.N. 1997: Curricular principles for health professions education about family violence. Academic Medicine 72, S51-58.

Britton, A. 2012: Intimate violence: 2010/11 BCS. In Smith, K., editor, Homicides, firearm offences and intimate violence 2010/11: supplementary volume 2 to crime in England and Wales 2010/11. London: Home Office, 83-118.

Coben, J.H. and Friedman, D.I. 2002: Health care use by perpetrators of domestic violence. The Journal of Emergency Medicine 22, 313-17.

Donovan, C. and Hester, M. 2014: Domestic violence and sexuality: what's love got to do with it? Bristol: Policy Press.

Feder, G., Davies, R.A., Baird, K., Dunne, D., Eldridge, S., Griffiths, C., Gregory, A., Howell, A., Johnson, M., Ramsay, J., Rutterford, C. and Sharp, D. 2011: Identification and Referral to Improve Safety (IRIS) of women experiencing domestic violence with a primary care training and support programme: a cluster randomised controlled trial. Lancet 378, 1788-95.

Gadomski, A.M., Wolff, D., Tripp, M., Lewis, C. and Short, L.M. 2001: Changes in health care providers' knowledge, attitudes, beliefs, and behaviors regarding domestic violence, following a multifaceted intervention. Academic Medicine 76, 1045-52.

Gutmanis, I., Beynon, C., Tutty, L., Wathen, C.N. and MacMillan, H.L. 2007: Factors influencing identification of and response to intimate partner violence: a survey of physicians and nurses. BMC Public Health 7, 12.

Hegarty, K., Taft, A. and Feder, G. 2008: Violence between intimate partners: working with the whole family. BMJ 337, a839.

Hester, M. 2013: Who does what to whom? Gender and domestic violence perpetrators in english police records. European Journal of Criminology 10, 623-37.

Hester, M., Westmarland, N., Gangoli, G., Wilkinson, M., O'Kelly, C., Kent, A. and Diamond, A. 2006: Domestic violence perpetrators: identifying needs to inform early intervention. Bristol: University of Bristol in association with the Northern Rock Foundation and the Home Office.

Hester, M., Williamson, E., Regan, L., Coulter, M., Chantler, K., Gangoli, G., Davenport, R. and Green, L. 2012: Exploring the service and support needs of male, lesbian, gay, bi-sexual and transgendered and black and other minority ethnic victims of domestic and sexual violence. Bristol: University of Bristol.

Hines, D.A. and Douglas, E.M. 2009: Women's use of intimate partner violence against men: prevalence, implications, and consequences. Journal of Aggression, Maltreatment \& Trauma 18, 572-86.

Hoare, J. and Jansson, K. 2008: Extent of intimate violence, nature of partner abuse and serious sexual assault, 2004/05, 2005/06 and 2006/07 British Crime Survey. In Povey, D., Coleman, K., Kaiza, P., Hoare, J. and Jansson, K., editors,

Primary Health Care Research \& Development 2015; 16: 281-288
British crime survey: homicides, firearm offences and intimate violence 2006/07 (supplementary volume 2 to crime in England and Wales 2006/7). London: Home Office Statistical Bulletin, 58-72.

Kimberg, L.S. 2008: Addressing intimate partner violence with male patients: a review and introduction of pilot guidelines. Journal of General Internal Medicine 23, 2071-78.

Lo Fo Wong, S., Wester, F., Mol, S.S. and Lagro-Janssen, T.L. 2006: Increased awareness of intimate partner abuse after training: a randomised controlled trial. British Journal of General Practice 56, 249-57.

McCaw, B., Berman, W.H., Syme, S.L. and Hunkeler, E.F. 2001: Beyond screening for domestic violence: a systems model approach in a managed care setting. American Journal of Preventive Medicine 21, 170-76.

Miller, D. and Jaye, C. 2007: GPs' perception of their role in the identification and management of family violence. Family Practice 24, 95-101.

Miller, D., Thow, N., Hall, J. and Martin, I. 2005: Documentation of family violence in New Zealand general practice. New Zealand Medical Journal 118, U1382.

National Institute of Clinical Excellence (NICE). 2014: PH50: domestic violence and abuse: how health services, social care and the organisations they work with can respond effectively. London: NICE.

Office for National Statistics (ONS). 2013: Violent crime and sexual offences, 2011/12. London: ONS: http://www.ons.gov. uk/ons/dcp171778_298904.pdf

Ramsay, J., Rutterford, C., Gregory, A., Dunne, D., Eldridge, S., Sharp, D. and Feder, G. 2012: Domestic violence: knowledge, attitudes, and clinical practice of selected UK primary healthcare clinicians. The British Journal of General Practice 62, e647-55.

RESPECT. 2010: Men's advice line call report 2010. London: RESPECT.

RESPECT. 2013: Principles of accreditation available from the respect web-site. Retrieved 1 November 2013 from http:// www.respect.uk.net/

Thompson, R.S., Rivara, F.P., Thompson, D.C., Barlow, W.E., Sugg, N.K., Maiuro, R.D. and Rubanowice, D.M. 2000: Identification and management of domestic violence: a randomized trial. American Journal of Preventive Medicine $19,253-63$.

Westmarland, N., Hester, M. and Reid, P. 2004: Routine enquiry about domestic violence in GP practices: a pilot study. Bristol: University of Bristol.

Williamson, E. 2000. Domestic violence and health: the response of the medical profession. Bristol: Policy Press.

Williamson, E. 2012: Measuring gender-based violence: issues of impact and prevalence. In Nakray, K., editor, Genderbased violence and public health: international perspectives on budgets and policies. London: Routledge.

Yeung, H., Chowdhury, N., Malpass, A. and Feder, G.S. 2012: Responding to domestic violence in general practice: a qualitative study on perceptions and experiences. International Journal of Family Medicine 2012, 960523, 1-7. 